\title{
Mental rotation of anthropoid hands: a chronometric study
}

L.G. Gawryszewski ${ }^{1}$, C.F. Silva-dos-Santos ${ }^{1}$, J.C. Santos-Silva ${ }^{1}$, A.P. Lameira ${ }^{1}$ and A. Pereira Jr. ${ }^{2}$

\author{
${ }^{1}$ Departamento de Neurobiologia, Universidade Federal Fluminense, \\ Niterói, RJ, Brasil \\ ${ }^{2}$ Departamento de Fisiologia, Universidade Federal do Pará, Belém, \\ PA, Brasil
}

\begin{abstract}
Correspondence
L.G. Gawryszewski

Departamento de Neurobiologia

UFF

Caixa Postal 100180

24001-970 Niterói, RJ

Brasil

Fax: +55-21-2629-2279

E-mail: gawryszewski_lg@ yahoo.com.br

Research supported by CNPq, FAPERJ, CAPES, PIBIC-PROPP-UFF, PROPP,

UFF, UFPA and FADESP.
\end{abstract}

Received April 28, 2006

Accepted January 5, 2007

\begin{abstract}
It has been shown that mental rotation of objects and human body parts is processed differently in the human brain. But what about body parts belonging to other primates? Does our brain process this information like any other object or does it instead maximize the structural similarities with our homologous body parts? We tried to answer this question by measuring the manual reaction time (MRT) of human participants discriminating the handedness of drawings representing the hands of four anthropoid primates (orangutan, chimpanzee, gorilla, and human). Twenty-four right-handed volunteers (13 males and 11 females) were instructed to judge the handedness of a hand drawing in palm view by pressing a left/right key. The orientation of hand drawings varied from $0^{\circ}$ (fingers upwards) to $90^{\circ}$ lateral (fingers pointing away from the midline), $180^{\circ}$ (fingers downwards) and $90^{\circ}$ medial (finger towards the midline). The results showed an effect of rotation angle $(F(3,69)=19.57, \mathrm{P}<0.001)$, but not of hand identity, on MRTs. Moreover, for all hand drawings, a medial rotation elicited shorter MRTs than a lateral rotation (960 and $1169 \mathrm{~ms}$, respectively, $\mathrm{P}<0.05)$. This result has been previously observed for drawings of the human hand and related to biomechanical constraints of movement performance. Our findings indicate that anthropoid hands are essentially equivalent stimuli for handedness recognition. Since the task involves mentally simulating the posture and rotation of the hands, we wondered if "mirror neurons" could be involved in establishing the motor equivalence between the stimuli and the participants' own hands.
\end{abstract}

The ability to mentally transform the relative position/configuration of objects in space is important when planning actions. Shepard and Metzler (1) published a seminal work describing the mental representation of physical objects. The authors investigated the conscious imagery of three-dimensional objects by measuring the manual reaction time (MRT) on a task in which human partici-
Key words

- Handedness recognition

- Mental rotation

- Manual reaction time

- Motor imagery

- Mirror neurons

- Imitation pants should decide whether two drawings displayed on a computer screen corresponded to the same object or not. In order to perform the test adequately, the participants had to mentally simulate the rotation of the objects. The results showed that the dynamics of mental rotation was similar to the rotation of real objects. For instance, the time necessary to complete the mental rotation increased 
with the rotation angle in a linear fashion.

Cooper and Shepard (2) extended their investigation to include drawings of human hands and studied the influence of stimulus orientation on handedness discrimination. The authors showed that the MRT of participants varied with the orientation angle of the stimuli, being shorter when the fingers pointed upwards $\left(0^{\circ}\right)$ and longer when they pointed downwards $\left(180^{\circ}\right)$. They concluded that participants mentally rotated the drawings to the vertical position before deciding on handedness. More recently, Parsons (3), using a slight variation of the protocol employed by Cooper and Shepard (2) showed that the MRT for handedness discrimination does not depend solely on the orientation of the pictures, but mainly on the difficulty in imagining our own hands assuming the position of the drawings. This conclusion arose from experiments comparing the MRT for handedness discrimination with the time spent by the participants to either move their

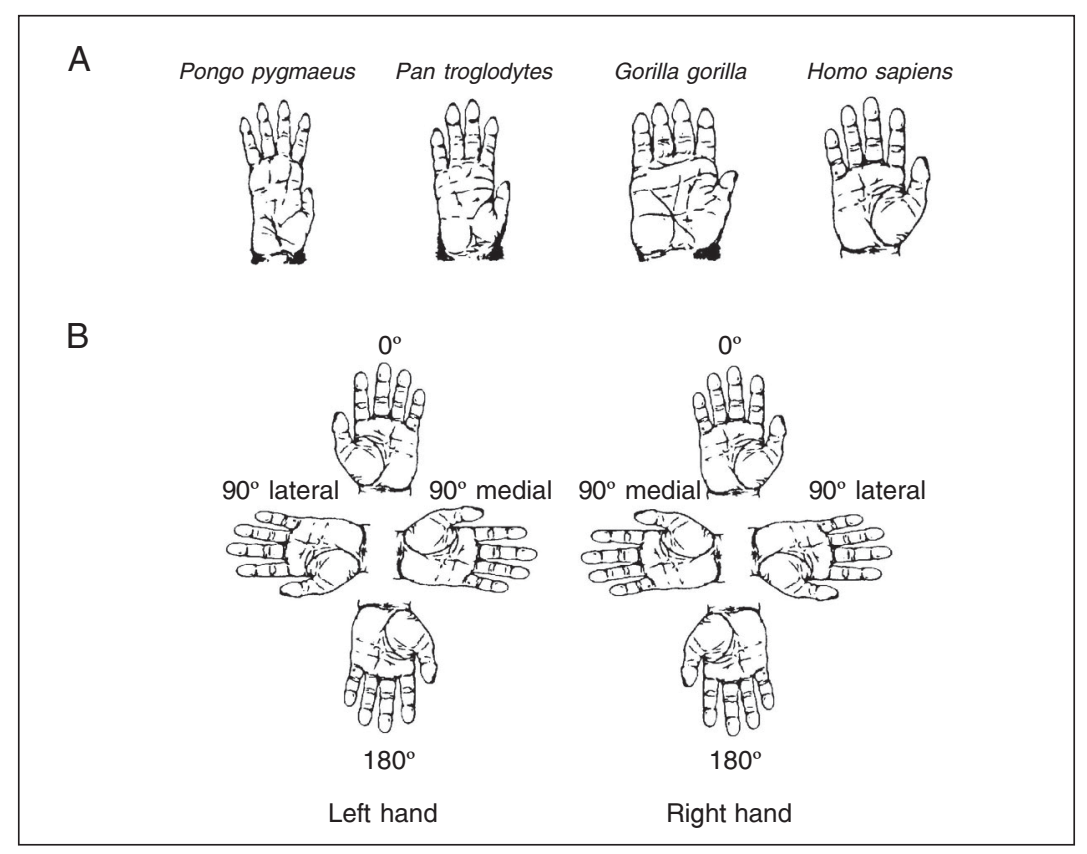

Figure 1. $A$, Drawings of the right hands of four anthropoid primates used in the experiment. $B$ (4 left hands), Drawings of the left human hand in the following orientations used in the experiment (counterclockwise): $0^{\circ}$ (fingers upwards), $90^{\circ}\left(90^{\circ}\right.$ lateral), $180^{\circ}$, and $270^{\circ}\left(90^{\circ}\right.$ medial); (4 right hands), drawings of the right human hand in the following orientations used in the experiment (clockwise): $0^{\circ}$ (fingers upwards), $90^{\circ}\left(90^{\circ}\right.$ lateral), $180^{\circ}$, and $270^{\circ}\left(90^{\circ}\right.$ medial) (adapted from Ref. 19). hands onto the stimulus configuration or just imagine this movement. Based on these findings, Parsons (3) proposed that the participants, instead of mentally rotating the drawings like non-self objects, performed a mental simulation of their own hand's moving to match the picture on the screen. Furthermore, this motor imagery was subject to the same biomechanical constraints imposed on the real movement, thus explaining the longer MRTs obtained with pictures of the hands in "awkward" positions (3).

In the present study, we evaluated the MRT of human participants discriminating the handedness of drawings of the hands of four anthropoid primates (orangutan, chimpanzee, gorilla, and human) presented in 4 orientations. Our aim was to determine whether the drawings belonging to non-human primates would be recognized as external, non-self objects, or whether they would be recognized as equivalent to the human hand. According to previous results, only in the last case would the MRT be sensitive to biomechanical constraints of the movement.

The experiments involved 24 righthanded (4) volunteers (13 males and 11 females, 18-39 years old, mean $=22.6$ years), with normal or corrected vision and naïve about the purposes of the experiment. Written informed consent was obtained from all volunteers and the experiment was approved by the Fluminense Federal University Ethics Committee. Drawings of hands (about $16^{\circ} \mathrm{x}$ $9^{\circ}$ ) of 4 anthropoid primates (orangutan, Pongo pygmaeus; chimpanzee, Pan troglodytes; gorilla, Gorilla gorilla, and human, Homo sapiens) were displayed on a computer screen (Figure 1A). The drawings were presented in the following orientations (Figure 1B): for the right hand $-0^{\circ}$ (fingers upwards), $90^{\circ}$ (clockwise - $90^{\circ}$ lateral), $180^{\circ}$, or $270^{\circ}\left(90^{\circ}\right.$ medial $)$ and for the left hand $-0^{\circ}$ (fingers upwards), $90^{\circ}$ (counterclockwise $90^{\circ}$ lateral $), 180^{\circ}$, or $270^{\circ}\left(90^{\circ}\right.$ medial $)$.

Experiments were carried out inside a sound-attenuated room, with a PC 486 com- 
puter used for both controlling the stimulus display and recording the participants' responses. Participants were positioned on a chin rest located $57 \mathrm{~cm}$ away from a 20" VGA computer monitor. The MEL 2.0 software was used to control the temporal sequence of events and to record MRTs. Stimuli were exhibited on the central region of the screen and the subject's task was to indicate handedness by pressing either a left or right switch.

All volunteers participated in only one experimental session, which consisted of a 32-trial training block and a full session of 320 trials divided into 4 blocks separated by rest periods of a few minutes. Each trial began with the presentation of a gray screen for $1000 \mathrm{~ms}$, followed by the display of a hand drawing, which remained onscreen until the subject responded by pressing a switch. Next, the gray screen was presented again for $500 \mathrm{~ms}$ before a new trial began. The trials with wrong or anticipatory (MRT $<100$ $\mathrm{ms}$ ) responses were repeated at the end of the session. During a session, the subject was instructed to keep his/her gaze fixed on the center of the screen and to respond as fast as possible to the occurrence of the stimulus.

The number of errors made by the 24 participants corresponded to only $2.3 \%$ (0$6.4 \%$ ) of the trials and those were not further analyzed. In addition, data from two participants were discarded due to the high number of errors (66 and 119 errors, respectively, corresponding to 17 and $27 \%$ of the trials).

The median MRT values were evaluated by a three-way repeated measure ANOVA with the following factors: angle $\left(0^{\circ}, 90^{\circ}\right.$ lateral, $180^{\circ}$ and $90^{\circ}$ medial), primate (orangutan, chimpanzee, gorilla, and human) and hand (left, right). The criterion for statistical significance was preset at $\alpha=0.05$.

The only factor with a significant effect on MRT was angle $(F(3,69)=19.57, \mathrm{P}<$ 0.001 ; Figure 2$)$. There was also no significant interaction among the factors. Thus, rotation angle influenced MRT irrespective of whether the hand depicted in the drawings belonged to a human or not.

Figure 2 shows the MRTs obtained for each hand orientation. The post hoc Newman-Keuls analysis showed that MRT was shorter $(\mathrm{P}<0.05)$ when fingers pointed upwards $\left(0^{\circ}-875 \mathrm{~ms}\right)$ than when fingers pointed both away from the midline $\left(90^{\circ}\right.$ lateral $1169 \mathrm{~ms})$ and downwards $\left(180^{\circ}-1299 \mathrm{~ms}\right)$, but did not differ $(\mathrm{P}>0.05)$ from the MRT obtained when fingers pointed towards the midline $\left(90^{\circ}\right.$ medial - $\left.960 \mathrm{~ms}\right)$. All other MRT comparisons were significantly different. It is worth noting that there was a significant difference between the MRT to the $90^{\circ}$ lateral $(1169 \mathrm{~ms})$ and $90^{\circ}$ medial $(960 \mathrm{~ms})$ orientations. This suggests that biomechanical constraints (3) must be influencing the mental rotation of drawings of both human and non-human hands.

Our main finding is the equivalence of MRTs for handedness discrimination of drawings representing the hands of both human and non-human primates. These results imply that the basic elements of motor imagery associated with this task (see Refs. 3 and 5) are similar in both cases. Our initial hypothesis was that the drawings of the hands of non-human primates would be treated like drawings of other non-self objects, i.e., there would be no difference on MRTs due

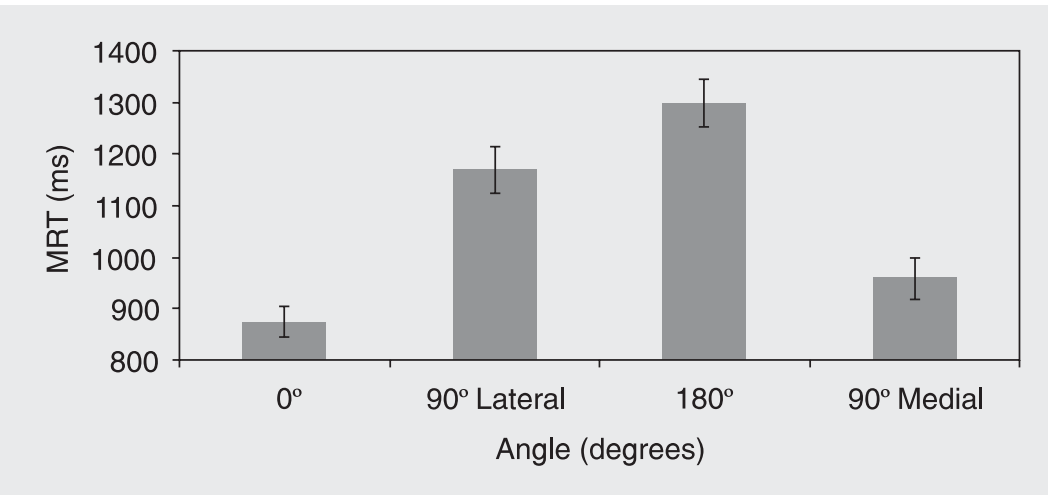

Figure 2. Manual reaction time (MRT) as a function of the orientation angle of the hand drawings. Notice that the MRT (mean \pm SEM) for the drawings with fingers pointing upwards $\left(0^{\circ}\right)$ is significantly shorter when compared to drawings with fingers pointing both away from the midline $\left(90^{\circ}\right.$ lateral) and downwards $\left(180^{\circ}\right)$, but not from drawings with fingers pointing towards the midline $\left(90^{\circ}\right.$ medial). Data are reported for 320 trials by 24 volunteers and the total number of trials was 7680 . 
to the lack of biomechanical constraints on motor imagery $(3,5)$. However, the results indicated just the opposite, as shown by the different MRTs for the $90^{\circ}$ lateral and $90^{\circ}$ medial postures (see above).

Parsons (3) had shown before that during performance of a handedness recognition task based on drawings of human hands the participants mentally simulated the movement of their own hand matching the picture displayed on the screen. According to Parsons (3), this motor imagery is subject to the same biomechanical constraints imposed on the real hand movement, thus explaining the longer MRTs obtained with pictures of hands displayed in "awkward" positions (3). In the present study, we showed that similar biomechanical restrictions seem to apply during handedness discrimination of non-conspecifics' hands, implying that in order to accomplish the task the observer also mentally simulates the movement of his/her own hand in a confirmatory fit. We suggest that the hand drawings of both humans and anthropoid primates are mapped onto the same motor representation in the brain, regardless of specific anatomical differences, because both stimuli engage the same preattentive mechanism responsible for the preliminary attribution of handedness (3,6-8, see below).

The mentally simulated movement used for handedness discrimination activates cortical areas involved with motor control in humans (for a review, see Ref. 9). More specifically, a PET study (9) showed that the pre-supplementary motor area, Brodmann areas (BA) 44, 46, and 4 in the left hemisphere and BA 6, 7, and 37 in the right hemisphere are involved in both motor imagery and handedness recognition.

Buccino and co-workers (10), in an fMRI study, showed that the same motor regions in the brain are activated when human participants observe an action present in their motor repertoire, such as biting, performed either by humans or monkeys. This mapping of an observed action onto the brain's motor representation may form the basis for action understanding. This hypothesis was introduced after the discovery of neurons located in area F5 of the monkey's premotor cortex that showed congruency between the visual actions they respond to and the motor behavior they specify $(6,7)$. The discharge of these "mirror neurons" generates an internal motor representation of the observed action, which is involved in the understanding of motor events $(6,7,11,12)$. Later, other areas in the monkey were shown to have the same properties, such as the superior temporal sulcus (STS) and area 7b, and were consolidated into a mirror neuron system (MNS) (for a review, see Ref. 8). Though neurons in extrastriate visual areas, such as the inferotemporal lobe and STS (8), also respond to hand and face stimuli (13, for reviews, see Refs. 14 and 15), and contribute to action understanding through perceptual mechanisms, they do not discharge during active self-movements.

Several sets of experimental data demonstrate that an MNS devoted to hand, mouth, and foot actions is also present in humans (10). The homologous MNS areas in humans are the STS, the inferior parietal lobe, and the ventral premotor cortex, including Broca's area $(8,16,17$, see Ref. 18 for a review). Moreover, an fMRI study (11) has shown that the observation of actions performed with the hand, the mouth and the foot leads to the activation of different sectors within these areas (BA 44 and 6), following a somatotopic pattern that resembles the classical motor cortex homunculus (11).

We would like to propose, as a tentative parsimonious explanation, that the handedness discrimination task we just described also engages the human MNS. More specifically, the particular orientation displayed by the hand drawing activates a corresponding subset of MNS neurons. Activation of this neuronal subpopulation would be responsible for pre-attentively triggering the implicit hand movement which precedes the 
conscious handedness decision $(3,5)$. The motor imagery associated with this implicit action is then mapped back onto the MNS, where it resonates with a stored motor representation and allows for the adequate handedness discrimination.

\section{Acknowledgments}

We dedicate this work to Professor Carlos Eduardo Rocha-Miranda (Academia Brasileira de Ciências), whose scientific achievements have inspired several generations of Brazilian neuroscientists.

\section{References}

1. Shepard RN, Metzler J. Mental rotation of three-dimensional objects. Science 1971; 171: 701-703.

2. Cooper LA, Shepard RN. Mental transformations in the identification of left and right hands. J Exp Psychol Hum Percept Perform 1975; 104: 48-56.

3. Parsons LM. Temporal and kinematic properties of motor behavior reflected in mentally simulated action. J Exp Psychol Hum Percept Perform 1994; 20: 709-730.

4. Oldfield RC. The assessment and analysis of handedness: the Edinburgh inventory. Neuropsychologia 1971; 9: 97-113.

5. Parsons LM. Integrating cognitive psychology, neurology and neuroimaging. Acta Psychol 2001; 107: 155-181.

6. Rizzolatti G, Fadiga L, Gallese V, Fogassi L. Premotor cortex and the recognition of motor actions. Brain Res Cogn Brain Res 1996; 3: 131-141.

7. Gallese V, Fadiga L, Fogassi L, Rizzolatti G. Action recognition in the premotor cortex. Brain 1996; 119 (Part 2): 593-609.

8. Rizzolatti G, Fogassi L, Gallese V. Neurophysiological mechanisms underlying the understanding and imitation of action. Nat Rev Neurosci 2001; 2: 661-670.

9. Parsons LM, Fox PT. The neural basis of implicit movements used in recognising hand shape. Cog Neuropsychol 1998; 15: 583-615.

10. Buccino G, Lui F, Canessa N, Patteri I, Lagravinese G, Benuzzi F, et al. Neural circuits involved in the recognition of actions performed by nonconspecifics: an fMRI study. J Cogn Neurosci 2004; 16: 114-126.

11. Binkofski F, Buccino $G$. The role of ventral premotor cortex in action execution and action understanding. J Physiol Paris 2006; 99: 396-
405.

12. Rizzolatti G, Fadiga L. The mirror-neuron system and action recognition. In: Freund HJ, Jeannerod M, Hallett M (Editors), Higher-order motor disorders: from Neuroanatomy and Neurobiology to Clinical Neurology. New York: Oxford University Press; 2005. p 141-157.

13. Gross CG, Rocha-Miranda CE, Bender DB. Visual properties of neurons in inferotemporal cortex of the Macaque. $J$ Neurophysiol 1972; 35: 96-111.

14. Tanaka K. Inferotemporal cortex and object vision. Annu Rev Neurosci 1996; 19: 109-139.

15. Pinsk MA, Desimone K, Moore T, Gross CG, Kastner S. Representations of faces and body parts in macaque temporal cortex: a functional MRI study. Proc Natl Acad Sci U S A 2005; 102: 69967001.

16. lacoboni M. Understanding others: Imitation, language and empathy. In: Hurley S, Chater N (Editors), Perspectives on imitation: From Neuroscience to Social Science. Volume 1: Mechanisms of imitation and imitation in animals (Social Neuroscience). Cambridge: MIT Press; 2005.

17. Rizzolatti G. The mirror neuron system and imitation. In: Hurley S, Chater N (Editors), Perspectives on imitation: From Neuroscience to Social Science. Volume 1: Mechanisms of imitation and imitation in animals (Social Neuroscience). Cambridge: MIT Press; 2005.

18. Aziz-Zadeh L, Koski L, Zaidel E, Mazziotta J, lacoboni M. Lateralization of the human mirror neuron system. J Neurosci 2006; 26: 29642970.

19. Schultz AH. The life of primates. New York: Universe Books; 1969. 